\title{
An intelligent light-managing ionic skin for UV-protection, IR stealth, and optical camouflaged Morse codes
}

\author{
Xiaofang Shi ${ }^{1,3}$, Zhouyue $\mathrm{Lei}^{\mathrm{i}^{*}}$ and Peiyi $\mathrm{Wu}^{1,3^{*}}$
}

\begin{abstract}
Ionic skins that demonstrate great advantages in the mechanical properties and multiple sensory capabilities are regarded as an attractive candidate to mimic functions of human skin. However, human skin is vulnerable to be damaged under long-time sunlight irradiation, and most of the current ionic skins also lack a protection against harmful ultraviolet and infrared lights. Herein, this work develops a multifunctional ionic skin based on ionic conductive and light-managing hydrogels via a facile one-step locally confined polymerization. It is mechanically adaptable, able to modulate light in the broadband solar spectrum, and protect human skin from the harmful ultraviolet and infrared lights. Moreover, without complicated processing, the ionic skin enables human-machine interactions via wireless and optical camouflaged Morse codes. We believe this work will promote the development of smart wearable devices with multiple customizable functions.
\end{abstract}

Keywords: ionic skins, hydrogels, light-managing, human-machine interfaces

\section{INTRODUCTION}

Human skin is a major organ, which serves as a physical barrier to protect inner body. It contains various sensory receptors to perceive ambient environment stimuli [1]. Recently, artificial skins with the advantages of mechanical adaptability and multiple signal sensory abilities have been developed to emulate the functions of human skin [2-5]. However, rare artificial skins are reported with the capability to protect human skin. Under a long-time exposure to sunlight, ultraviolet (UV) light induces the concentrating of melanin which may cause skin cancers [6], and infrared (IR) light generates interior heat and may cause overheating of skin [7]. Therefore, it is imperative to develop light-managing artificial skins that can protect human skin from the harmful wavelengths of sunlight.

For the light modulation purpose, artificial skins require electrochromic [8], thermochromic [9,10], or photochromic [11] fillers with tunable transmittance to manage UV, visible, and IR lights [12,13]. Meanwhile, as a bridge to connect human and machines, light-managing artificial skins require good con- ductivity and sensory functions. To combine multiple functions in an artificial skin system, it usually requires multi-layer assembly technologies, which results in a high cost of production $[14,15]$. Moreover, mechanical rigidity of inorganic fillers shortens the service life of artificial skins and renders them nonrecyclable. Therefore, to realize light management and integrate multifunction in artificial skins, it is critical to develop organic materials with customizable functions via energy-efficient and environmentally sustainable ways $[16,17]$.

Herein, this work develops intelligent ionic skins based on polyelectrolyte hydrogels via a facile one-step locally confined polymerization (LCP) to combine broadband light management capability and relatively high ionic conductivity $[9,18]$. The LCP is similar to self-emulsifying polymerization. There is a liquidliquid phase separation during the polymerization, which could be tuned by hydrogen bonding and hydrophobic associations between the monomer and dispersion medium [19]. The LCPgenerated hierarchical nano-micro structures are advantageous for broadband light management [9]. The hydrogen bonding and hydrophobic associations in the final hydrogel enable not only widely tunable thermochromic function but also relatively high modulus, large stretchability, and self-healing for reuses $[9,20]$. Furthermore, the free ions in the polyelectrolyte hydrogel could act as charge carriers for information transmission without the need for additional processing of conductive layers. The ionic skin is optically camouflaged and the relatively high ionic conductivity enables intelligent dialogue via wireless international Morse codes. The material design and working mechanism are also discussed in detail, which is expected to inspire the development of smart wearable displays with multiple customizable functions.

\section{EXPERIMENTAL SECTION}

\section{Materials}

Methacrylic acid (MAA), ammonium persulfate (APS), methylene blue, and $\mathrm{D}_{2} \mathrm{O}$ were obtained from Sigma-Aldrich Co. Quaternary chitosan (QCS) was purchased from Nantong Lvshen Bioengineering Co., Ltd. Fluorescein sodium, methylene blue, and photoinitiator 2959 were purchased from Thermo Fisher Scientific.

\footnotetext{
${ }^{1}$ State Key Laboratory for Modification of Chemical Fibers and Polymer Materials, College of Chemistry, Chemical Engineering and Biotechnology, Center for Advanced Low-Dimension Materials, Donghua University, Shanghai 201620, China

2 John A. Paulson School of Engineering and Applied Sciences, Harvard University, Cambridge, MA, 02138, USA

${ }^{3}$ State Key Laboratory of Molecular Engineering of Polymers, Department of Macromolecular Science, Fudan University, Shanghai 200433, China

* Corresponding authors (emails: zhouyuelei@seas.harvard.edu (Lei Z), peiyiwu@fudan.edu.cn (Wu P))
} 
Preparation of QCS-poly(MMA) (PMAA) hydrogel (QMAAH)

Firstly, the QCS was dissolved in deionized water at different concentrations with magnetic stirring at room temperature. Then $20 \mathrm{wt} \%$ MAA monomer and APS with different molar ratios (compared with MAA) were added to the solution. After mixing and degassing, the solution was heated at $70^{\circ} \mathrm{C}$ for $6 \mathrm{~h}$.

\section{Preparation of PMAA hydrogel}

The PMAA was prepared by initiating the polymerization of $20 \mathrm{wt} \%$ MAA monomer aqueous solution with $0.2 \mathrm{~mol} \%$ APS at $70^{\circ} \mathrm{C}$ for $6 \mathrm{~h}$.

\section{Preparation of the samples for the time-dependent Fourier transform infrared spectroscopy (FTIR) analysis}

The time-dependent FTIR absorbance spectrum of the LCP process was recorded to analyze the molecular dynamics during the LCP process. In order to eliminate the overlap of $\mathrm{H}_{2} \mathrm{O}$ at around $1640 \mathrm{~cm}^{-1}, \mathrm{D}_{2} \mathrm{O}$ instead of $\mathrm{H}_{2} \mathrm{O}$ was used as the solvent. The samples with different polymerization times were obtained by sampling during the QMAAH preparation process every two minutes and quenching the reaction in an ice bath and exposing in air.

\section{UV-shielding evaluation}

A piece of QMAAH/PMAA film (1 mm in thickness) was prepared in a polyethylene (PE) culture dish with a diameter of $5 \mathrm{~cm}$. The solution used for the photocatalytic degradation test was composed of $0.001 \mathrm{wt} \%$ methylene blue and $0.2 \mathrm{~mol} \%$ photoinitiator 2959. The solution was injected into two $2-\mathrm{mL}$ glass bottles and covered by the PE culture dish containing QMAAH film and PMAA film respectively. The opening and side parts of the culture dishes were protected by tinfoil paper. The dishes were then exposed to $365-\mathrm{nm}$ UV light simultaneously for 10 and $30 \mathrm{~min}$. The transmittance of methylene blue exposed to UV for a specified time was detected at $200-800 \mathrm{~nm}$.

\section{Characterization}

The UV-vis spectrum was measured with a Lamda $35 \mathrm{UV}$-Vis (Shimadzu, Japan) spectrophotometer. The hydrogel was sandwiched in a glass cell with a 1-mm-thick slit. The turbidity as a function of temperature was detected at $500 \mathrm{~nm}$ with PMAA hydrogel as the reference. The temperature was controlled by a water-jacketed cell holder with a heating rate of $1^{\circ} \mathrm{C} \mathrm{min}^{-1}$.

The FTIR transmittance spectra were recorded on a Thermo Fisher spectrometer (Nicolet 6700, USA) in the range of 2.5$25 \mu \mathrm{m}$. The temperature-dependent FTIR absorbance spectra were detected with a temperature interval of $1^{\circ} \mathrm{C}$. The hydrogel was sealed in two calcium fluoride tablets. Two-dimensional (2D) correlation spectroscopy FTIR analysis was performed by the software of 2D Shige, ver. 1.3 (@Shigeaki Morita, Kwansei Gakuin University, Japan, 2004-2005).

The frequency-dependent storage modulus $\left(G^{\prime}\right)$ and loss modulus $\left(G^{\prime \prime}\right)$ of the hydrogel were conducted on a HAAKE MARS III rheometer with a panel fixture and a Peltier heating/ cooling element. The frequency curves were generated by extension of frequency sweeps from 0.1 to $10 \mathrm{~Hz}$ in the temperature range from 15 to $35^{\circ} \mathrm{C}$. The temperature intervals were $5^{\circ} \mathrm{C}$ and the shift factors were obtained with a reference temperature of $20^{\circ} \mathrm{C}$.

The strain-stress curves were tested on a universal mechanical test machine (Instron 5966, USA). The resistance signals were recorded on a portable test system (LinkZill ACR-MP-01, Hangzhou). The applied voltage was supplied by a TH6203 direct-current (DC) power supply.

Ionic conductivity measurements were carried out on a CHI660E electrochemical workstation via DC polarization experiments. Field emission scanning electron microscopy (FESEM) image was recorded by Zeiss Ultra 55 . The micrographs of the polymerization process of the hydrogel were observed by a microscope (Leica DM2500P, Germany).

\section{RESULTS AND DISCUSSION}

\section{Material design of the light-managing ionic skin}

In this work, amphiphilic MAA is used as the monomer, while QCS and APS synergistically provide rich ions to modulate the LCP. Without APS, pure MAA aqueous solution (20 wt $\%$ ) and QCS aqueous solution (5.0 wt\%) are homogenous and transparent because of the dominant hydration of carboxyl and quaternary ammonium groups (Fig. S1a). For the MAA solution, when the concentration of APS is higher than $7.3 \mathrm{~mol} \%$, microphase separation occurs (Fig. S1b). Interestingly, for the mixed aqueous solution of MAA (20 wt $\%)$ and QCS (1.0 wt \%), a small quantity of APS $(1.0 \mathrm{~mol} \%)$ could trigger the microphase separation (Fig. 1a). This is probably because the introduction of APS not only provides ions but also induces the chain entanglement of QCS, resulting in higher hydrophobicity of the precursor [21,22]. Therefore, the QCS and APS synergistically promote the microphase separation of the monomer precursor solution which is important to tune the LCP process [9]. Moreover, the QCS as a natural polysaccharide, plays an important role in mechanical optimization due to the chain entanglement with the PMAA via electrostatic interactions [22,23].

During the LCP process, the liquid-liquid phase separation gradually disappears, probably due to the difference in hydrophilic/hydrophobic properties of the monomer and final polymer (Fig. 1b). The obtained hydrogel is free-standing, highly transparent, capable of being reshaped at room temperature, and compliable on the skin surface (Fig. 1c). The molecular reaction kinetics of the LCP process was further studied by FTIR (Fig. 1d). The characteristic peak located at $1630 \mathrm{~cm}^{-1}$ belongs to the carbon-carbon double bond of the MAA monomer. This characteristic peak disappears gradually in $14 \mathrm{~min}$, indicating the completion of LCP. The characteristic peak of $\mathrm{v}\left(\mathrm{CH}_{3}\right)$ and $\mathrm{v}\left(\mathrm{CH}_{2}\right)$ located at $2860-3060 \mathrm{~cm}^{-1}$ shifts gradually to a higher frequency, indicating the hydrophobic groups of the reaction solution are gradually hydrated with the proceeding of LCP. Therefore, the liquid-liquid phase separation disappears and the resulting hydrogel is hydrophilic enough to keep transparent in visible light at room temperature. As observed by FESEM (Fig. 1e), the morphology of the hydrogel is composed of micron-sized networks with high-density sidewalls and nanoscale networks located inside. This result further confirms the formation of hierarchical networks (Fig. 1f).

Widely tunable transparency in visible light region and multiscale structure evolution analysis

Without chemical crosslinkers, the hydrogel prepared via LCP is physically crosslinked and the nano-micro structure is dynamic and tunable upon stimuli. For example, although the hydrogel is transparent in visible region at room temperature, it could 


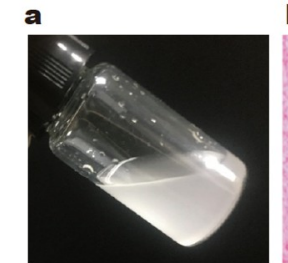

b
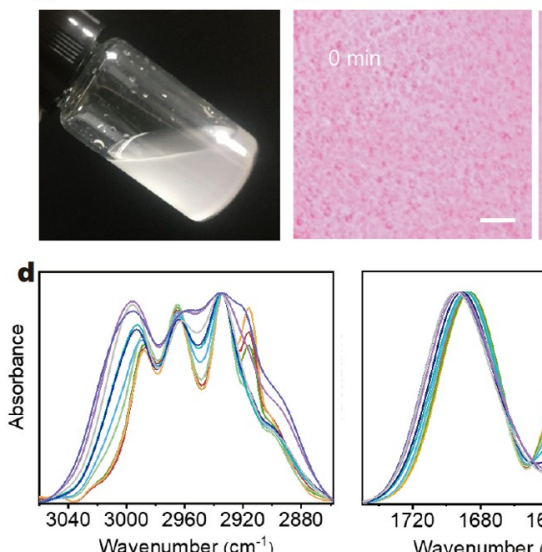

Wavenumber $\left(\mathrm{cm}^{-1}\right)$

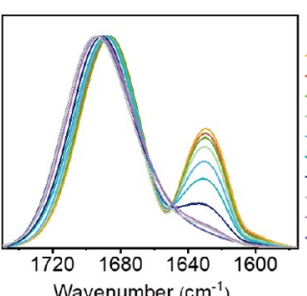

Wavenumber $\left(\mathrm{cm}^{-1}\right)$

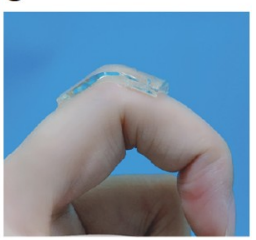

f
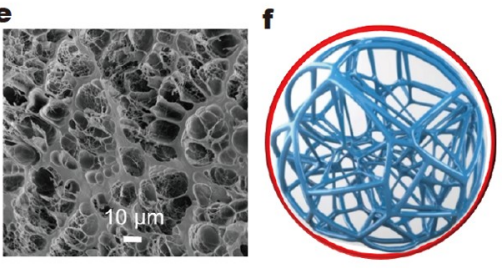

Figure 1 (a) A photograph of the microphase-separated precursor solution. (b) The optical micrographs of the precursor solution during the LCP process. The scale bars are $100 \mu \mathrm{m}$. (c) A photograph of QMAAH attached to human skin. (d) The normalized time-dependent FTIR results of the precursor solution during the LCP process. (e) The FESEM image of the freeze-dried QMAAH. (f) A schematic model of the hierarchical structure of the QMAAH.
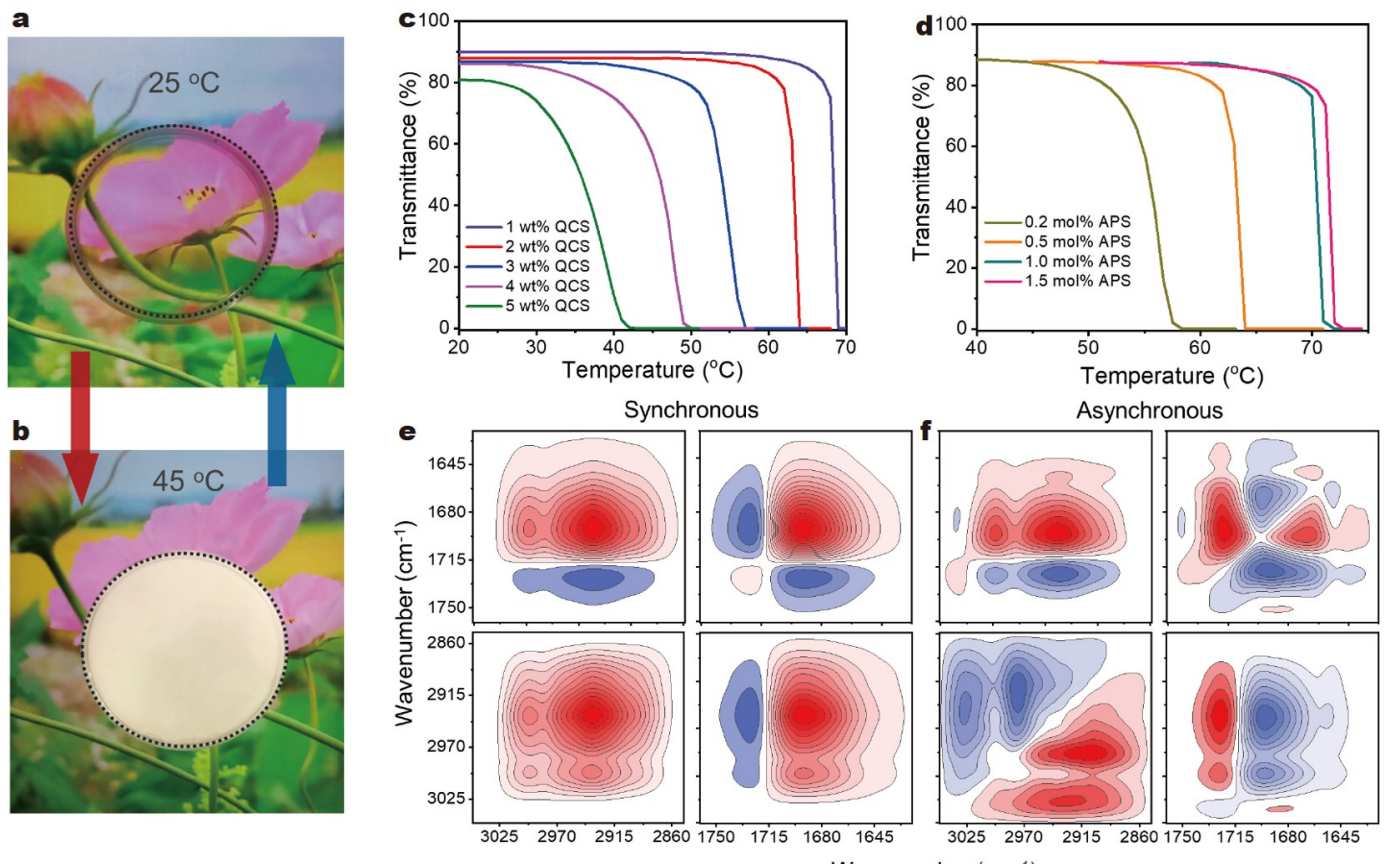

Wavenumber $\left(\mathrm{cm}^{-1}\right)$

Figure 2 ( $\mathrm{a}, \mathrm{b})$ Photograph record the reversible temperature phase transition behavior of QMAAH. (c) Transmittance vs. temperature curves for the QMAAH with different QCS concentrations. (d) Transmittance vs. temperature curves for the QMAAH with different APS concentrations. (e) The 2D synchronous spectra of the QMAAH during the phase transition. (f) The 2D asynchronous spectra of the QMAAH during the phase transition. In the 2D correlation spectra, the blue areas represent negative correlation intensity, while the red colors indicating positive intensity.

become optically opaque at higher temperatures. The volume phase transition temperature (VPTT) and transparency of QMAAH depend on the concentrations of QCS and APS. The transmittance of QMAAH in the visible band reversibly switches upon temperature changing around VPTT. The VPTT of QMAAH with the feeding amount of 5.0\% QCS and $0.5 \mathrm{~mol} \%$ APS is $36^{\circ} \mathrm{C}$ (Fig. 2a, b). When keeping the concentration of APS at $0.5 \mathrm{~mol} \%$, the VPTT of QMAAH decreases with the increase of QCS content (Fig. 2c). This is ascribed to the increased hydrophobicity of the QMAAH with the increasing ionic strength. On the other hand, when the concentration of QCS remains unchanged at $2.0 \mathrm{wt} \%$, the VPTT of QMAAH increases with the increase of APS content. This is probably because the ammonium ion of APS can associate with the carboxyl of MAA; the ratio of ammonium ion and persulfate ions in the molecular formula of APS is 2:1, thus the general ionic strength decreases at the relatively small amount of APS (Fig. 2d). When the concentration of APS is above $1.0 \mathrm{~mol} \%$, the increasing total ion strength weakens the influence of APS on VPTT. A diagram summarizes the relationship between the VPTT of QMAAH and the concentration of QCS/APS (Fig. S2). The results suggest that the transmittance of QMAAH in the visible region can be widely tuned by changing the feeding ratio of QCS and APS. The increase of ionic strength leads to the increase of hydrophobicity and the decrease of VPTT and vice versa. When the usage of QCS is higher than $4.0 \mathrm{wt} \%$ with the presence of $0.2 \mathrm{~mol} \%$ APS, 
the QMAAH is opaque under visible light due to the dominant hydrophobic effect of QCS and PMAA chains.

2D correlation FTIR spectroscopy further reveals the working mechanism of the QMAAH [24]. QMAAH with a VPTT of $36^{\circ} \mathrm{C}$ is taken as an example. The molecular interactions in the QMAAH network, including hydrophobic a-methyl and methylene chain segments and hydrophilic carboxylic acid groups, were investigated to explore the dynamic structural evolution of QMAAH during phase transition (Fig. 2e, f) [25]. The bands located at $1760-1610$ and $3050-2850 \mathrm{~cm}^{-1}$ belong to $\mathrm{v}(\mathrm{C}=\mathrm{O})$ of the carboxylic acid group and $\mathrm{v}(\mathrm{C}-\mathrm{H})$ of the $\alpha-$ methyl group and the main chain, respectively. Overall, the slight red shifting of the bands $\mathrm{v}(\mathrm{C}-\mathrm{H})$ and $\mathrm{v}(\mathrm{C}=\mathrm{O})$ observed in the temperature-dependent FTIR spectra (Fig. S3) implies the dehydration of the chemical groups [26,27]. On the basis of 2D synchronous and asynchronous spectra (Fig. 2e, f), the specific sequence order of different chemical groups changing upon heating is defined as $\mathrm{v}(\mathrm{CH})\left(2908 \mathrm{~cm}^{-1}\right) \rightarrow \mathrm{v}\left(\mathrm{CH}_{2}\right)\left(2929 \mathrm{~cm}^{-1}\right)$ $\rightarrow \mathrm{v}\left(\right.$ associated $\left.\mathrm{CH}_{3}\right) \quad\left(3027 \mathrm{~cm}^{-1}\right) \rightarrow \mathrm{v}\left(\right.$ disassociated $\left.\mathrm{CH}_{3}\right)$ $\left(2976 \mathrm{~cm}^{-1}\right) \rightarrow \mathrm{v}($ associated $\mathrm{COOH})\left(1668 \mathrm{~cm}^{-1}\right) \rightarrow \mathrm{v}($ disassociated $-\mathrm{COOH})\left(1691 \mathrm{~cm}^{-1}\right) \rightarrow \mathrm{v}($ associated $-\mathrm{C}=\mathrm{O} \ldots \mathrm{OH}-)$ $\left(1697 \mathrm{~cm}^{-1}\right) \rightarrow \mathrm{v}($ disassociated $-\mathrm{C}=\mathrm{O} \ldots \mathrm{O}=\mathrm{C}-)\left(1722 \mathrm{~cm}^{-1}\right)$. This order infers that the phase transition begins with the motions of $\mathrm{CH}$ groups and $\mathrm{CH}_{2}$ groups belonging to the QCS main chain and PMAA main chain, respectively, and then drives the dehydration of the pendant $\alpha$-methyl groups of PMAA. Subsequently, these changes lead to further disruptions of the intrachain hydrogen bond between the carbonyl group of PMAA chains and the hydroxyl of QCS chains. Finally, the disassociation of the hydrogen bonding among the PMAA chains occurs in the heating process. These results indicate that polymer chains firstly undergo a conformation change in the phase transition process, which is probably driven by the aggregation of chitosan chains during heating [23]. Temperature-dependent in-situ small-angle X-ray scattering (SAXS) results reveal the evolution of delicate structures below $100 \mathrm{~nm}$. As shown in Fig. S4a, in the high $q$ region $\left(q>0.9 \mathrm{~nm}^{-1}\right)$, the scattering curves of QMAAH $\left(\right.$ VPTT $=52^{\circ} \mathrm{C}$ ) only have small changes with the temperature rising. The result means that the structure of the molecular level does not change with temperature rising. In the low $q$ region, the intensity of the scattering curve increases with the increase of temperature below $57^{\circ} \mathrm{C}$. The intensity of the scattering curve becomes weaker with the increase of temperature above $57^{\circ} \mathrm{C}$. The scattering intensities were fitted by the correlation length model (Fig. S4b, more details are discussed in Theory models in the Supplementary information). At the $q$ regime, the correlation length $\xi$, which implies the mesh size of the nano-network, increases from several nanometers to a dozen nanometers up to $57^{\circ} \mathrm{C}$. This is because as the temperature increases, the molecular chains agglomerate to form a thicker skeleton structure. The thickening of the skeleton means that the average distance of the porous structure skeleton becomes larger.

\section{UV-shielding, infrared stealth, and heat insulation}

Besides the tunable optical transmittance in visible region, the LCP-prepared hydrogel with the dynamic nano-micro structure could modulate the transmittance in UV and even IR regions. The electromagnetic spectrum of UV is located at $10-400 \mathrm{~nm}$ and can be subdivided into three regions named UVC (100$280 \mathrm{~nm}), \operatorname{UVB}(280-315 \mathrm{~nm})$, and UVA (315-400 nm) [28]. Over exposure to UV radiation can cause the accumulation of melanin in human skin. Especially, the UVA can penetrate the dermis and even trigger skin cancer. The effective components of existing commercial sunscreen are mainly $\mathrm{TiO}_{2}$ or $\mathrm{ZnO}$, which can sufficiently shield UVB and UVC but are not satisfied for UVA due to their large optical band gaps [28,29]. In this work, the nanoscale networks in QMAAH block UV light via the physical process. Meanwhile, the active groups in QCS further improve the UV shielding ability of QMAAH via ionic interactions with PMAA [30]. The synergy effects endow the QMAAH with excellent light managing ability in the UV region. The thickness of the QMAAH ionic skin utilized for the UV protection test is about $1 \mathrm{~mm}$. When the sunlight is irradiated on the QMAAH ionic skin, the UV light is scattered and absorbed by the nanoscale networks in QMAAH and the QCS chains, while the visible light penetrates through the ionic skin. For the pure PMAA hydrogel without the addition of QCS and nanoscale networks, it can hardly block the UV light above $250 \mathrm{~nm}$. For the QMAAH prepared in QCS aqueous solutions with different concentrations, the UV light below $365 \mathrm{~nm}$ was almost entirely blocked (Fig. 3a and Fig. S5). Especially, more than 95\% of the UVA below $365 \mathrm{~nm}$ can be blocked. The efficient shielding of UV light was further confirmed by the UV protection test. As shown in Fig. 3b, a piece of square-shaped QMAAH was attached to the surface of fresh porcine skin and exposed to UV light. To prevent dehydration, the porcine skin was covered with a plastic wrap during the UV irradiation process. After $2 \mathrm{~h}$ irradiation, the naked porcine skin was tanned. While the area protected by QMAAH skin maintained the same appearance before the UV irradiation. It suggests an excellent UV blocking effect of the QMAAH skin. Furthermore, methylene blue was used for a photocatalytic degradation test. As a photodegradable material, methylene blue can be easily degraded when exposed to 365-nm UVA light in the presence of a photoinitiator. As shown in Fig. S6a, the methylene blue solution covered by QMAAH could be effectively protected from degradation, but the one covered by PMAA was faded from blue to almost colorless within $30 \mathrm{~min}$. Fig. S6b shows the absorption spectra of methylene blue solution exposed to UVA light at different times. The characteristic absorption peaks of methylene blue (665 and $610 \mathrm{~nm}$ ) almost disappeared within $30 \mathrm{~min}$ for the solution protected by the PMAA film. However, the absorption peaks of the solution protected by QMAAH film kept almost unchanged during the irradiation process. To this end, the QMAAH shows a massive potential in the applications of protecting the skin from UV damage. Besides, the QMAAH-based ionic skin shows an IR stealth and heat insulation effect. As shown in Fig. 3c, double PE slides are semi-transparent in the range of $2.5-25 \mu \mathrm{m}$. In contrast, a piece of $50-\mu \mathrm{m}$ QMAAH sandwiched between the PE slides can block the IR totally in the range of $2.5-25 \mu \mathrm{m}$. We attribute the excellent IR light-blocking property of QMAAH to the micro-networks whose dimensionless size parameter is included in the range of the IR band. Furthermore, the IR light blocking effect is not influenced by the phase transition process under visible light. The QMAAH can successfully block the broadband IR and manage lights in the whole solar spectrum. In light of the excellent thermal IR blocking effect and sunlightinduced tunable transparency, the QMAAH can be further utilized as a smart ionic skin for IR stealth and heat insulation. The thermal radiation of objects is located in the IR band and can be detected by a thermal IR camera. As shown in Fig. 3d, a human hand can be detected with a higher temperature (about $32^{\circ} \mathrm{C}$ ) 

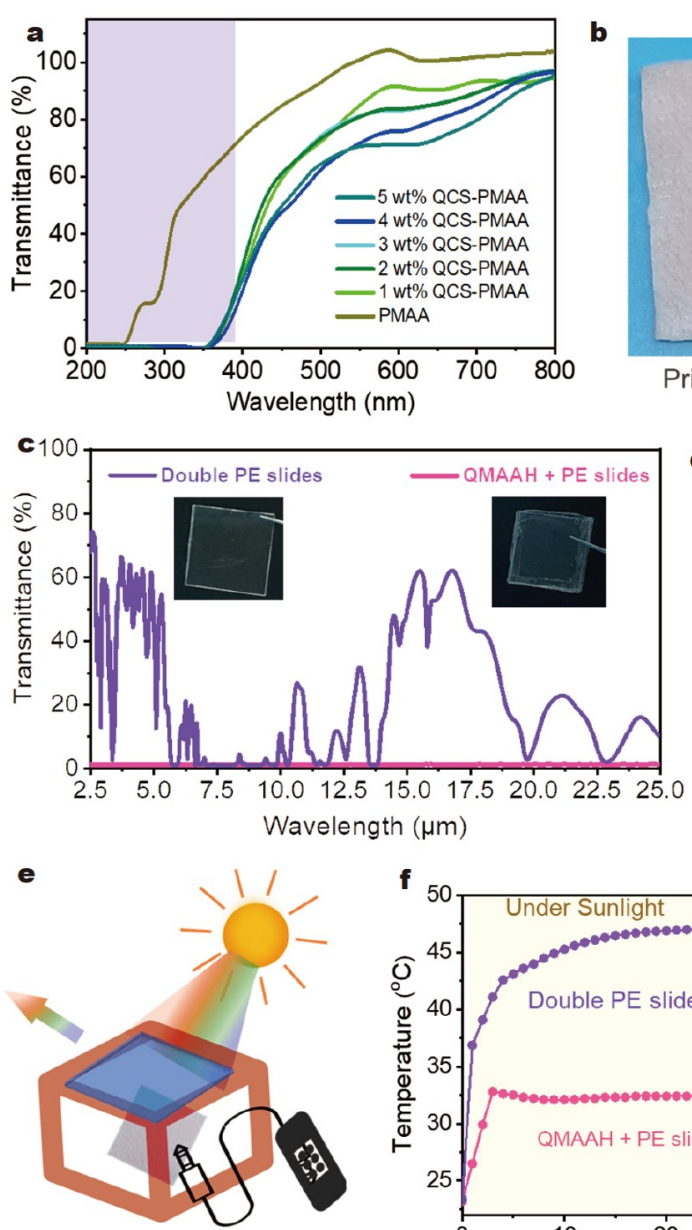

b

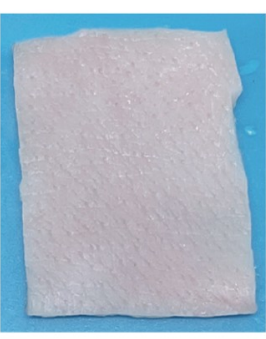

Pristine Porcine skin

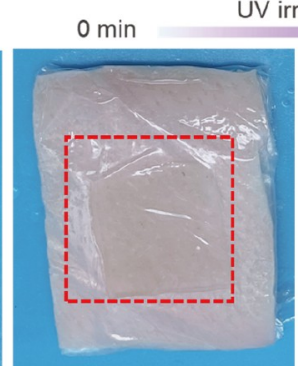

Protected by QMAAH

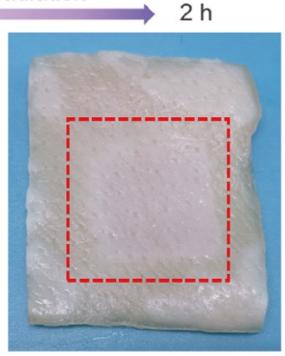

After UV irradiation
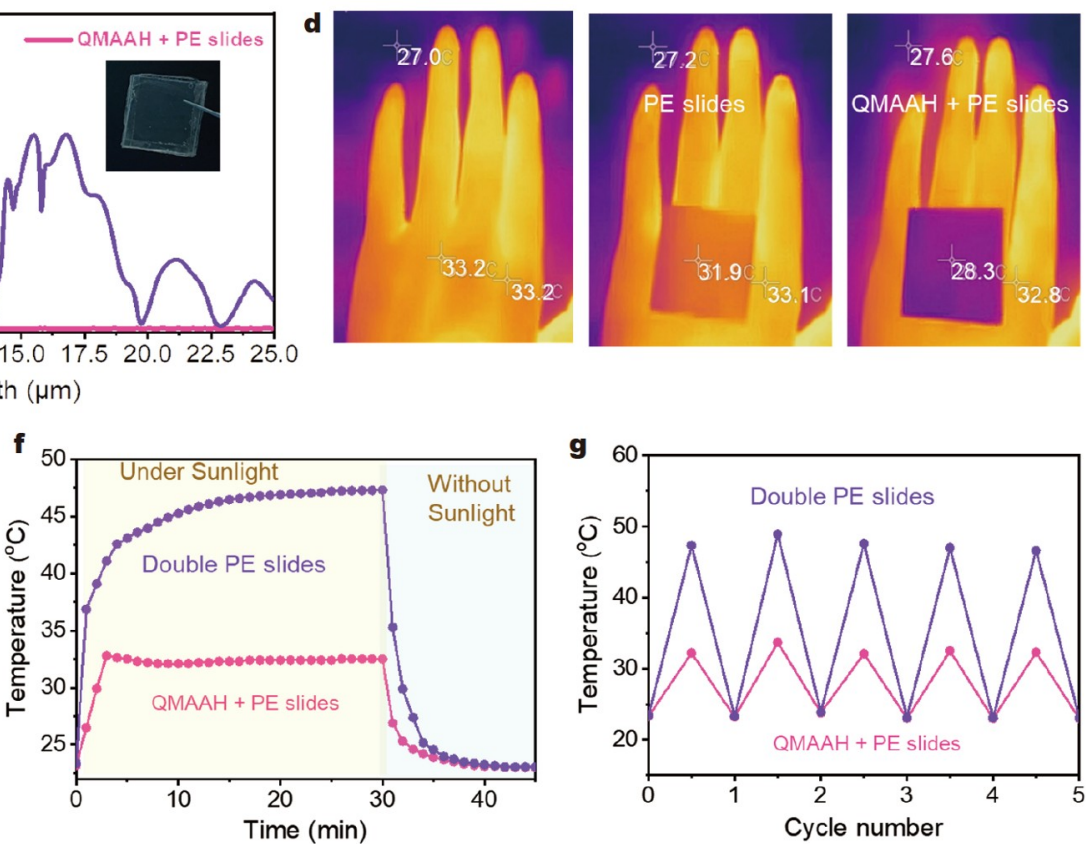

Figure 3 (a) UV-vis transmittance spectra of the QMAAH ionic skin with various QCS contents. (b) UV shielding test of the QMAAH ionic skin on a piece of fresh porcine skin. (c) The transmittance results of double PE slides and QMAAH ionic skin in the IR band. (d) The thermal IR image of a naked human hand (left) and the corresponding images of the hand covered by double PE slides (middle) and the QMAAH ionic skin (right). (e) Schematic illustration of a model chamber used for the heat insulation test. The surfcace temperature of porcine skin placed in the chamber was recorded by a thermocouple. (f) Temperature vs. time profiles of the thermocouple extended to the interior model chamber with the PE slides or QMAAH as the roof. (g) Cyclic temperature records of the thermocouple extended to the interior model chamber assembled with different roofs.

than the ambient environment (about $27^{\circ} \mathrm{C}$ ). The PE slides covered on the hand can be easily detected by an IR camera due to poor IR blocking ability. In contrast, the QMMAAH skin can entirely block the IR light and the temperature was similar to the ambient environment (about $28.4^{\circ} \mathrm{C}$ ), making the covered area stealth from the detection of the IR camera. To further evaluate the heat insulation effect of the QMAAH skin, a thermal insulation chamber was installed with the double $\mathrm{PE}$ slides or QMAAH as a roof (Fig. 3e). A piece of porcine skin was placed inside the chamber and its surface temperature was recorded by a thermocouple. As shown in Fig. 3f, when the chamber assembled with QMAAH skin was irradiated by sunlight for $3 \mathrm{~min}$, the temperature increased from 23 to $33^{\circ} \mathrm{C}$. With the irradiation time increased, the QMAAH skin switched from a transparent to an opaque state. The inner temperature kept stable at about $32.5^{\circ} \mathrm{C}$. The chamber assembled with PE slides was used for a parallel test. The temperature increased sharply to about $41^{\circ} \mathrm{C}$ within $3 \mathrm{~min}$ and kept increasing to a plateau of about $47^{\circ} \mathrm{C}$, which was $14.5^{\circ} \mathrm{C}$ higher than that of the QMAAH skin. When the chambers were removed away from sunlight after $30 \mathrm{~min}$, the inner temperature decreased to $23^{\circ} \mathrm{C}$ in $10 \mathrm{~min}$. The cyclic tests further confirm the stable solar energy shielding ability of the QMAAH skin (Fig. 3g).

\section{Mechanical robustness and self-healing of the QMAAH}

The hierarchical networks can reinforce the mechanical property of hydrogels [9]. In this work, the cationic QCS can not only provide ionic strength to induce the formation of hierarchical networks via LCP but also act as rigid chains to enhance the mechanical strength of QMAAH [31]. Besides, the strong ionic interaction between QCS and MAA can also contribute to the reinforcement of hydrogel networks. As shown in Fig. $4 \mathrm{a}$, in the frequency sweep measurements of the storage $\left(G^{\prime}\right)$ and loss $\left(G^{\prime \prime}\right)$ moduli, the QMAAH with a VPTT of $36^{\circ} \mathrm{C}$ shows a solid-like behavior in the temperature range of $15-35^{\circ} \mathrm{C}$. The apparent activation energy is calculated from the slope of the curve in Fig. $4 \mathrm{~b}$. On the basis of the time-temperature equivalence principle of the Arrhenius equation, the apparent activation energy is calculated to be $160 \mathrm{~kJ} \mathrm{~mol}^{-1}$. This value is far higher than those of traditional supramolecular networks (41- 

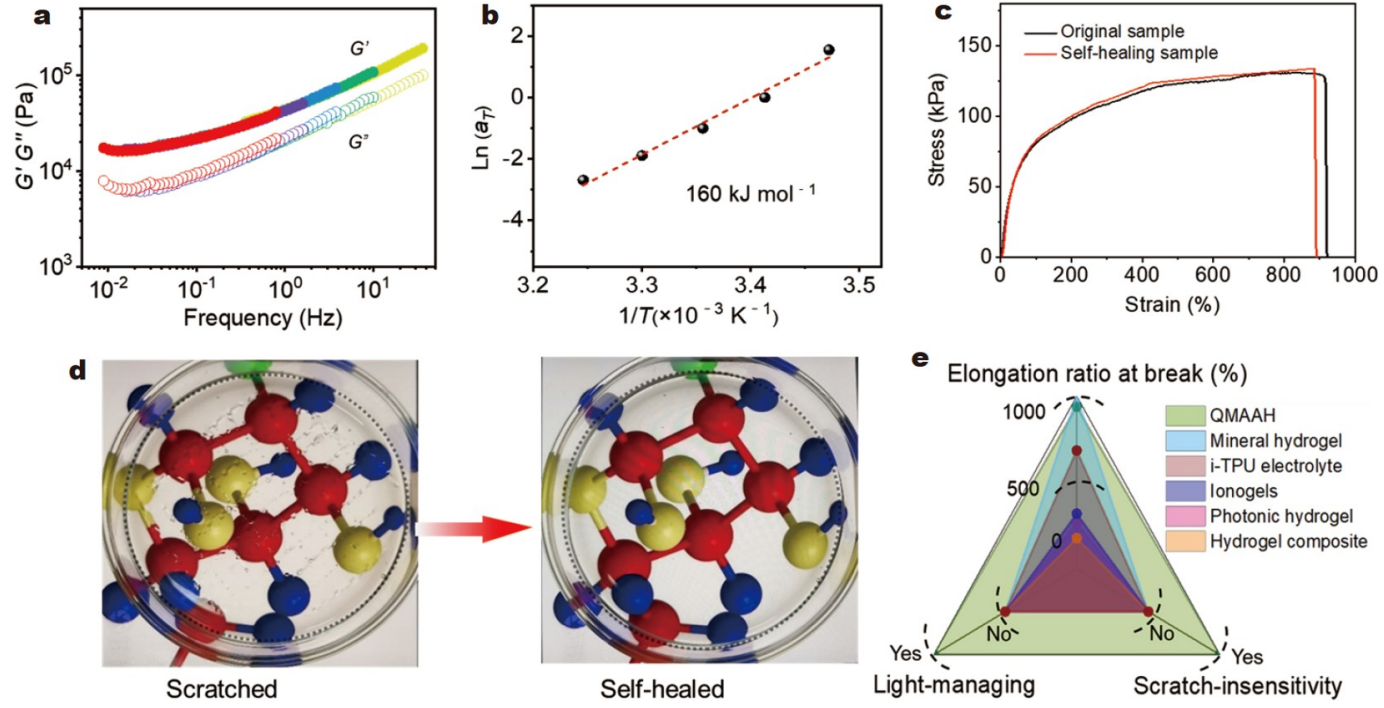

Figure 4 (a) Frequency-dependent storage modulus $\left(G^{\prime}\right)$ and loss modulus $\left(G^{\prime \prime}\right)$ of the QMAAH. This master curve is generated by shifting and superimposing different frequency sweeping curves in a temperature range of 15 to $30^{\circ} \mathrm{C}$. (b) Arrhenius plot depicting the temperature dependence of shift factor $\left(a_{\mathrm{T}}\right)$. (c) The strain-stress curve of the original QMAAH and the self-healing one. (d) Photographs of the QMAAH with scratches and the self-healed sample (The hydrogel is outlined by dotted line). (e) A critical comparison about the self-protection functions among the QMAAH and previous reported organic ionic skins based on mineral hydrogel [35], ionic thermoplastic polyurethane (i-TPU) electrolyte film [36], ionogels [37], photonic hydrogel [38] and hydrogel composite [39].

$96 \mathrm{~kJ} \mathrm{~mol}^{-1}$ ) [32]. The high energy barriers of QMAAH trap polymer chains and enable mechanical robustness. The QMAAH could be stretched, twisted, and knotted (Fig. S7). As shown in Fig. 4c, the Young's modulus of hierarchical-structure QMAAH is calculated to be $182 \mathrm{kPa}$ and the elongation ratio at break is $920 \%$, which is better than those of the chemically crosslinked networks $[33,34]$. Furthermore, due to the reversible physically cross-linked networks (including $\mathrm{H}$-bonding, ionic interaction, hydrophobic association, and chain entanglement), the QMAAH possesses excellent self-healing ability. The severed QMAAH sample can recover its mechanical properties as the original sample within $48 \mathrm{~h}$ of self-healing. Especially, the QMAAH exhibits insensitivity towards repeated scratches. The apparent scratch on the QMAAH can be almost repaired within several minutes (Fig. 4d), the scratch-insensitivity behavior of QMAAH is provided in Movie S1 (Supplementary information). A critical comparison about the self-protection functions between the QMAAH ionic skin and previous reported organic ionic skins, including mineral hydrogel [35], electrolyte film [36], ionogels [37], photonic hydrogels [38], and hydrogel composite [39], is provided in Fig. 4e. Most of the organic ionic skins are limited in mechanical intensity, easy to scrape and unable to modulate broad-band sunlight. In contrast, the QMAAH ionic skin shows a wide range of advantages, combining robustness, scratch-insensitivity, and light management.

\section{Wireless human-machine interactions of the ionic skin}

The ionic conductivity of the QMAAH with a VPTT of $36^{\circ} \mathrm{C}$ was measured and calculated to be $0.52 \mathrm{~S} \mathrm{~m}^{-1}$ (Note S1, Fig. 5a). In light of the relatively high ionic conductivity, the QMAAH was further exploited as a sensory platform for wireless humanmachine interactions. As illustrated in Fig. 5b, a QMAAH ionic skin was constructed by sandwiching a piece of QMAAH film between two pieces of PE slides. When the ionic skin was subjected to an external pressure, the resistance of the dynamic hydrogel networks changed, which induced the circuit out of balance, and a voltage was output proportional to the pressure. According to Ohm's law, the increase of voltage results in the corresponding resistance increase at a constant current. Human beings can encrypt what they want to "say" by pressing the QMAAH skin rhythmically to generate visual resistance signals in the form of international Morse codes [40]. The encrypted information can be wirelessly transformed from the detector terminal to a smartphone with real-time feedback (Fig. 5c). In the principle of the international Morse codes, all the alphabets are defined with different permutations and a combination of "dots" and "dashes" (Fig. 5d). For practical applications, the "dots" and "dashes" are transformed into visual resistance signals in real-time. For instance, the warning signals of "TAKE CARE" (Fig. 5e) and the distress signals of "HELP ME" (Fig. 5f) were recoded by pressing the QMAAH skin rhythmically with different duration times. As shown in Movie S2, the signals can be received by a smartphone via wireless transmission technology in real time. A picture of the portable test system is provided in Fig. S8. The ionic skin also shows superior combination of stretchability and sensitivity. Moreover, it could sense the cyclic strain variation upon stretching to $50 \%$ strain with high stability (Fig. S9).

The QMAAH ionic skin can block UV light, stealth under thermal IR band, tune the transmittance in visible light upon heating for heat insulation. The broadband light management is of great significance for the application of ionic skin in some special environments, such as military stealth and confidential information transmission, communication in extreme environments with strong UV light.

\section{CONCLUSION}

In summary, this work develops a multifunction light-managing ionic skin with widely tunable light-modulating function and wireless human-machine interactions. It can block UV light to 

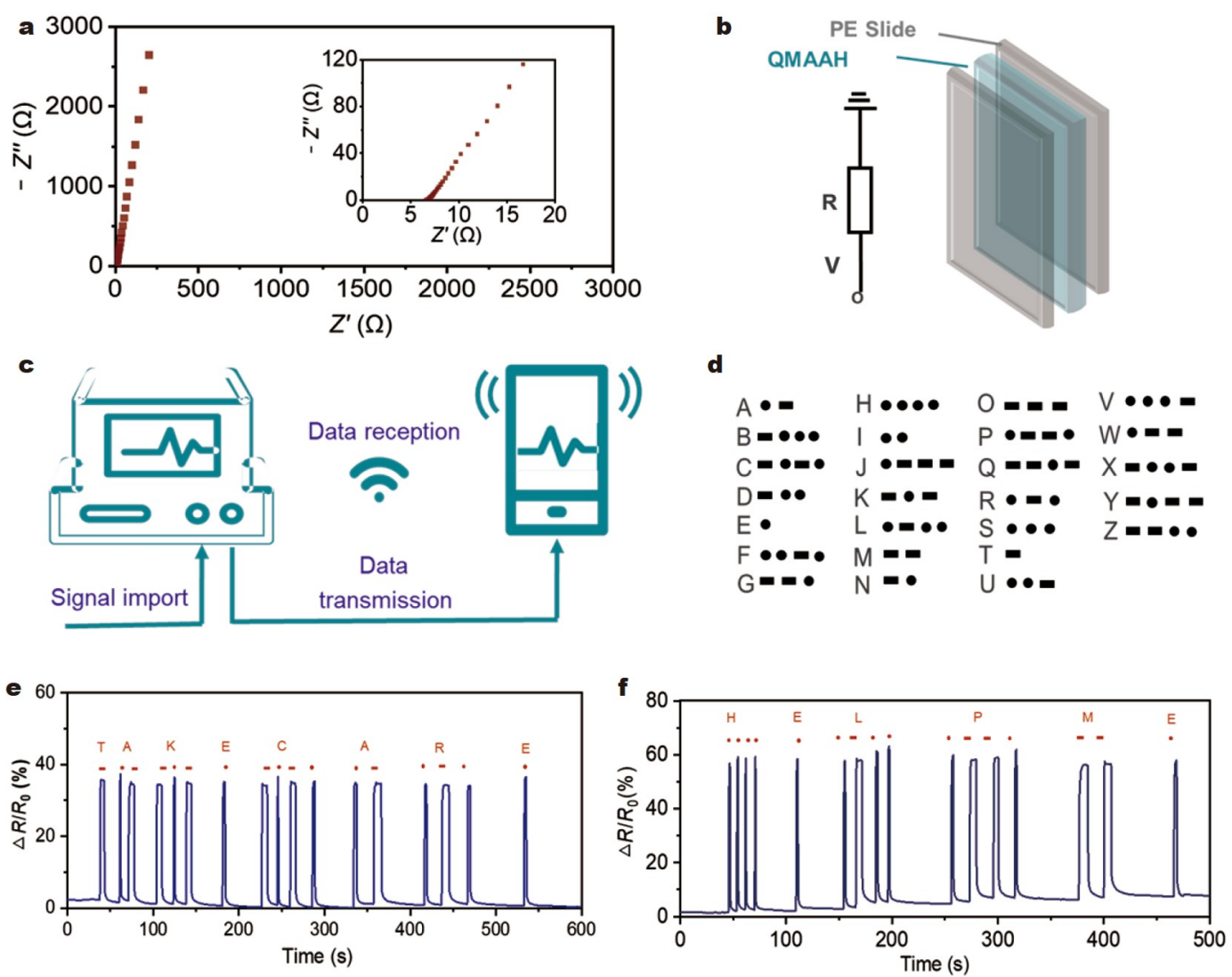

Figure 5 (a) The electrochemical impedance spectroscopy measurement of the QMAAH ionic skin. The inset is the magnified picture. (b) Schematic illustration of the QMAAH ionic skin sensor. (c) Schematic illustration of the wireless transmission system. (d) The related symbols of the international Morse codes in the alphabet. (e) The practical applications of QMAAH to recode and transmit the warning signal of "TAKE CARE". (f) The practical applications of QMAAH to recode and translate the distress signal of "HELP ME".

protect skin from damage, shield thermal IR light for stealth, dynamically tune the transmittance under solar light for heat insulation, and enables intelligent dialogue in the form of international Morse codes between humans and machines. In particular, the main function of this smart skin can be achieved by only a single layer of QMAAH, avoiding the need for complicated processing and reducing the cost for large-scale practical applications. Besides, the QMMAH ionic skins show impressive mechanical performance, including robustness, self-healing and scratch-insensitivity, which can increase the service life of ionic skins. We believe this work not only provides light-managing capability for ionic skin but may also promote the development of smart wearable ionic skins with multiple customizable functions.

Received 21 June 2021; accepted 6 September 2021; published online 13 October 2021

1 Sun JY, Keplinger C, Whitesides GM, et al. Ionic skin. Adv Mater, 2014, 26: 7608-7614

2 Lei Z, Zhu W, Zhang X, et al. Bio-inspired ionic skin for theranostics. Adv Funct Mater, 2021, 31: 2008020

3 Yang C, Suo Z. Hydrogel ionotronics. Nat Rev Mater, 2018, 3: 125-142

4 Chortos A, Liu J, Bao Z. Pursuing prosthetic electronic skin. Nat Mater, 2016, 15: 937-950

5 Shi X, Wu P. A smart patch with on-demand detachable adhesion for bioelectronics. Small, 2021, 17: 2101220

6 Wen J, Tang J, Ning H, et al. Multifunctional ionic skin with sensing, UV-filtering, water-retaining, and anti-freezing capabilities. Adv Funct
Mater, 2021, 31: 2011176

7 Khandelwal H, Schenning APHJ, Debije MG. Infrared regulating smart window based on organic materials. Adv Energy Mater, 2017, 7: 1602209

8 Yang P, Sun P, Mai W. Electrochromic energy storage devices. Mater Today, 2016, 19: 394-402

9 Lei Z, Wu B, Wu P. Hierarchical network-augmented hydroglasses for broadband light management. Research, 2021, 2021: 1-12

10 Zhang Q, Jiang Y, Chen L, et al. Ultra-compliant and tough thermochromic polymer for self-regulated smart windows. Adv Funct Mater, 2021, 31: 2100686

11 Pardo R, Zayat M, Levy D. Photochromic organic-inorganic hybrid materials. Chem Soc Rev, 2011, 40: 672-687

12 Shen X, Du J, Sun J, et al. Transparent and UV blocking structural colored hydrogel for contact lenses. ACS Appl Mater Interfaces, 2020, 12: 39639-39648

13 Lyu J, Liu Z, Wu X, et al. Nanofibrous kevlar aerogel films and their phase-change composites for highly efficient infrared stealth. ACS Nano, 2019, acsnano.8b08913

14 Wan C, Chen G, Fu Y, et al. An artificial sensory neuron with tactile perceptual learning. Adv Mater, 2018, 30: 1801291

15 Pang Q, Lou D, Li S, et al. Smart flexible electronics-integrated wound dressing for real-time monitoring and on-demand treatment of infected wounds. Adv Sci, 2020, 7: 1902673

16 Wang M, Luo Y, Wang T, et al. Artificial skin perception. Adv Mater, 2021, 33: 2003014

17 Yang JC, Mun J, Kwon SY, et al. Electronic skin: Recent progress and future prospects for skin-attachable devices for health monitoring, robotics, and prosthetics. Adv Mater, 2019, 31: 1904765

18 Gao W, Lei Z, Wu K, et al. Reconfigurable and renewable nano-microstructured plastics for radiative cooling. Adv Funct Mater, 2021, 31: 


\section{5}

19 Lovell PA, Schork FJ. Fundamentals of emulsion polymerization. Biomacromolecules, 2020, 21: 4396-4441

20 Lei $\mathrm{Z}, \mathrm{Wu}$ P. Zwitterionic skins with a wide scope of customizable functionalities. ACS Nano, 2018, 12: 12860-12868

21 Rinaudo M. Chitin and chitosan: Properties and applications. Prog Polym Sci, 2006, 31: 603-632

22 Yang Y, Wang X, Yang F, et al. A universal soaking strategy to convert composite hydrogels into extremely tough and rapidly recoverable double-network hydrogels. Adv Mater, 2016, 28: 7178-7184

23 Duan J, Liang X, Cao Y, et al. High strength chitosan hydrogels with biocompatibility via new avenue based on constructing nanofibrous architecture. Macromolecules, 2015, 48: 2706-2714

24 Noda I. Generalized two-dimensional correlation method applicable to infrared, Raman, and other types of spectroscopy. Appl Spectrosc, 1993, 47: $1329-1336$

25 Noda I. Two-dimensional infrared spectroscopy. J Am Chem Soc, 1989, 111: 8116-8118

26 Watanabe A, Morita S, Ozaki Y. Study on temperature-dependent changes in hydrogen bonds in cellulose i $\beta$ by infrared spectroscopy with perturbation-correlation moving-window two-dimensional correlation spectroscopy. Biomacromolecules, 2006, 7: 3164-3170

27 Cheng H, Shen L, Wu C. LLS and FTIR studies on the hysteresis in association and dissociation of poly( $N$-isopropylacrylamide) chains in water. Macromolecules, 2006, 39: 2325-2329

28 Ye B, Yao C, Yan M, et al. Photo-induced hydrogel formation based on g- $\mathrm{C}_{3} \mathrm{~N}_{4}$ nanosheets with self-cross-linked 3D framework for UV protection application. Macromol Mater Eng, 2018, 304: 1800500

29 Cardillo D, Sencadas V, Devers T, et al. Attenuation of UV absorption by poly(lactic acid)-iron oxide nanocomposite particles and their potential application in sunscreens. Chem Eng J, 2021, 405: 126843

30 Kowalonek J. Studies of chitosan/pectin complexes exposed to UV radiation. Int J Biol Macromolecules, 2017, 103: 515-524

31 You J, Xie S, Cao J, et al. Quaternized chitosan/poly(acrylic acid) polyelectrolyte complex hydrogels with tough, self-recovery, and tunable mechanical properties. Macromolecules, 2016, 49: 1049-1059

32 Lei Z, Wu P. A supramolecular biomimetic skin combining a wide spectrum of mechanical properties and multiple sensory capabilities. Nat Commun, 2018, 9: 1134

33 Sun JY, Zhao X, Illeperuma WRK, et al. Highly stretchable and tough hydrogels. Nature, 2012, 489: 133-136

34 Liu X, Liu J, Lin S, et al. Hydrogel machines. Mater Today, 2020, 36: 102-124

35 Lei Z, Wang Q, Sun S, et al. A bioinspired mineral hydrogel as a selfhealable, mechanically adaptable ionic skin for highly sensitive pressure sensing. Adv Mater, 2017, 29: 1700321

36 Jin ML, Park S, Kim JS, et al. An ultrastable ionic chemiresistor skin with an intrinsically stretchable polymer electrolyte. Adv Mater, 2018, 30: 1706851

37 Li T, Wang Y, Li S, et al. Mechanically robust, elastic, and healable ionogels for highly sensitive ultra-durable ionic skins. Adv Mater, 2020, 32: 2002706

38 Wang Y, Cao X, Cheng J, et al. Cephalopod-inspired chromotropic ionic skin with rapid visual sensing capabilities to multiple stimuli. ACS Nano, 2021, 15: 3509-3521

39 Yin XY, Zhang Y, Xiao J, et al. Monolithic dual-material 3D printing of ionic skins with long-term performance stability. Adv Funct Mater, 2019, 29: 1904716

40 Wang $\mathrm{H}$, Li S, Wang Y, et al. Bioinspired fluffy fabric with in situ grown carbon nanotubes for ultrasensitive wearable airflow sensor. Adv Mater, 2020, 32: 1908214

Acknowledgements This work was supported by the National Natural Science Foundation of China (51973035 and 51733003).

Author contributions Shi $\mathrm{X}, \mathrm{Wu} \mathrm{P}$ and Lei $\mathrm{Z}$ designed the experiments. Shi $\mathrm{X}$ performed the experiments and prepared the manuscript. Wu $\mathrm{P}$ and Lei $\mathrm{Z}$ supervised the project and revised the manuscript.
Conflict of interest The authors declare that they have no conflict of interest.

Supplementary information Experimental details and supporting data are available in the online version of the paper.

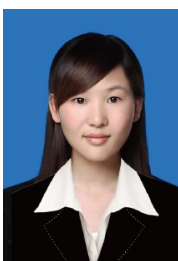

Xiaofang Shi received her $\mathrm{PhD}$ degree in polymer chemistry and physics under the supervision of Prof. Peiyi $\mathrm{Wu}$ at Fudan University (2021). Her research interest mainly focuses on the syntheses and application of multifunctional hydrogel materials.

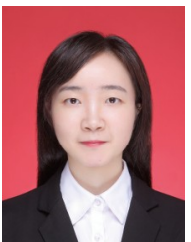

Zhouyue Lei is a postdoctoral fellow at Harvard University. She received her $\mathrm{PhD}$ degree in polymer chemistry and physics under the supervision of Prof. Peiyi Wu at Fudan University (2019). Her research interest mainly focuses on soft ionic materials and their intelligent applications.

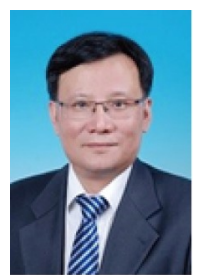

Peiyi Wu is a professor at the College of Chemistry, Chemical Engineering and Biotechnology, Donghua University (China) and a fellow of the Royal Society of Chemistry. He received his $\mathrm{PhD}$ degree at the University of Essen (Germany) in 1998. His research interests are focused on the 2D-IR spectroscopy, flexible hydrogel electronics and iontronics, and the synthesis and application of $2 \mathrm{D}$ materials.

\section{“光调控”的智能水凝胶离子皮肤用于紫外线防护、 红外屏蔽和光学伪装的莫尔斯电码}

时晓芳 1,3 , 雷周玥 $2^{*}$, 武培怡 $1,3^{*}$

摘要 由于广泛可调的机械性能和多重感应功能, 离子皮肤被认为是 模拟人体皮肤功能的最具吸引力的候选材料. 然而, 目前报道的离子皮 肤大多数缺乏对太阳光谱的管理能力以及对人体皮肤的光学保护作 用. 特别是长期暴露在太阳光下的皮肤很容易被紫外线辐射和红外光 引起的过热损伤. 本研究使用限域聚合的方法, 开发了一种温敏性的导 电聚合物水凝胶离子皮肤. 这种离子皮肤无需复杂的制备技术, 不仅能 够以国际莫尔斯电码的形式实现光学伪装的人机无线对话, 还可以对 全太阳光范围内的光谱进行调控, 并具有强韧的机械性能. 我们相信, 该离子皮肤的设计灵感也将启发其他具有可定制功能的智能可穿戴器 件的发展. 